\title{
Optimal Power Control and Time Allocation for Wireless- powered Relay Networks
}

\author{
Lin Xiao, Fahui Wu and Dingcheng Yang \\ Information engineering School, Nanchang University \\ xiaolin910@gmail.com,fahui97@126.com,676862214@qq.com
}

\begin{abstract}
In this letter, optimal power control and time resource allocation schemes are considered in wireless relay network, which two users communicate with each other assisted by an energy harvesting relay that gathers energy from the received signal by applying a time switching scheme and forwards the received signal by using the harvesting energy. It is focused on the optimal power control and time resource allocation for the system to cooperate the power transfer and information transmission among three nodes in Decode-and-Forward (DF) mode. Specifically, time ratios for three transmission phases are firstly investigated. Then, we proposed to formulate the power optimization problem with energy constraint. Since the transmission duration of the transmitter is not fixed, the energy constraint instead of power constraint is more reasonable to evaluate the system performance. Through some variables substitution, we demonstrate that it is a convex problem. It can be solved efficiently by convex optimization technology. Simulation results are demonstrated that the performance of the proposed algorithm outperform than the fixed and semi-fixed traditional methods.
\end{abstract}

Keywords: Energy cooperation; simultaneous information and power transfer; relay; power control.

\section{Introduction}

Energy harvesting is a promising technology to provide wireless networks with sustainable power supplies. It can overcome the bottleneck of energy constrained wireless networks. The nodes with energy harvesting technology can collect the different kinds of energy from the environment (wind/solar/tide energy) and convert it into usable electric power for the system. However, these energy sources always are affected by the weather, position, time and so on. It has intermittent and unpredictable nature and is critical for applications where the quality of service is of paramount importance [1].

Wireless power transfer technology, where the receiver can scavenge energy from the transmitted radio signal, has recently attracted much attention in academia and industry [2-4]. Simultaneous wireless information and power transfer is very convenient for mobile terminals, because it makes use of the RF signals in two ways: for transferring power as well as information. RF energy transfer can be fully controlled and therefore can be used for scenarios with strict QoS. Moreover, it can reduce the cost of communication networks, since peripheral equipment to take advantage of external energy sources can be avoided. This novel technology provides an exciting solution to meet the requirement for green communication.

The concept of wireless-powered is first proposed by Varshney in [5]. In [6], Zhou proposed the SWIPT architecture for receiver design and suggested two methods to distinguish information and energy: Time Switching (TS) and Power Splitting (PS). The work in [7] proposes an amplify-and-forward relaying protocol for SWIPT with TS and PS mode, and investigate the effect of different parameters on the system performance. In 
[8], the outage probability for a decode-and-forward (DF) cooperative network with TS mode is analyzed. Ding proposed several power allocation strategies to optimize the outage probability in DF cooperative network where multiple source-destination pairs communicate via a shared energy harvesting relay [9]. In [10], Krikidis investigated the role of relaying node in energy harvesting system.

In this letter, we focus on the optimal power control and time resource allocation issues in a simple three nodes with DF cooperation networks. Since the transmission duration for the transmitter is flexible, we propose to formulate the optimization problem with energy constraint to investigate the relationship of time ratios between power transfer and information transmission in DF cooperative network. Since it is a causal problem for power control and time resource allocation, the optimization problem is non-convex. We proposed a method to convert the joint optimization problem into a convex problem using the variable substitution. Despite the original problem is intractable, we construct an efficient algorithm to find an optimal solution with low computational complexity. Simulation results show that the proposed method can get higher throughput of the system, compared with the performance of optimal solutions in traditional protocols.

This letter is organized as follows: Section II introduces the system model and the basic notation. In Section III, we present the optimization problem and our proposed method. Simulation results and comparisons are given in Section IV, and finally, conclusions are drawn in Section V.

\section{System Model}

We consider an energy harvesting cooperative network with two users and a relay node as shown in Figure 1.



Figure 1. System Model

All nodes operate in half-duplex mode with single antenna. The transmit user is equipped with fix power supply. Two users cannot communicate with each other directly. They exchange the messages assisted by the energy harvesting relay node. The relay node is battery-free. It can scavenge the energy from its observation. It means that the relay node harvests the energy from RF signals transmitted by the transmitter and use this energy to relay transmitter's information.

The three-phase DF relaying protocol with time switching scheme for energy harvesting can be stated as follows:

In the first phase (Energy harvesting phase, EH phase), the source terminal $S_{a}$ transmits the signals to the energy harvesting relay node for energy transfer. It is worth noting that the EH receiver at the relay node does not need to convert the received signal from the RF band to the baseband for scavenging the carried energy. Nevertheless, according to the law of energy conservation, it can be supposed that the total harvested RF-band power is proportional to that of the received baseband signal. The harvested energy can be normalized by the baseband symbol period at 
the EH receiver. Therefore, the scavenged power at the relay node can be presented as:

$$
Q_{R}=\alpha_{1} \zeta P_{1}|h x|^{2}
$$

where $x$ denote the data symbol to be transmitted by source user, $h$ denotes the channel coefficients between the relay and the source terminals; $\zeta(0<\zeta<1)$ is the energy conversion efficiency at the relay node $R . \alpha_{1}$ denotes the percentage of transmission time allocated to the energy transfer time phase. For simplicity, it is assumed in (1) that the scavenged energy due to the background noise at the EH receiver is negligible and thus can be ignored.

In the second phase (Information decoding phase, ID phase), the source terminal $S_{a}$ transmits the signal to the energy harvesting relay node for information decoding. The relay's detection is based on the following observation:

$$
y_{R}=\sqrt{P_{2}} h x+n_{R}
$$

where $y_{R}$ denotes the received signal at the relay node. $n_{R}$ is the additional Gaussian noise for the baseband signal. The throughput of the system within the second phase can be expressed as:

$$
r_{R}=\alpha_{2} \log _{2}\left(1+P_{2}|h|^{2}\right)
$$

where $\alpha_{2}$ is the information transmission duration of the transmitter in the second phase. It is supposed that the channel coefficients during the first and second phase is static. $P_{2}$ is the transmit power for transmitter during the second phase. We consider a DF relay protocol for the relaying network with energy harvesting. The relay node will decode the signal and reconstruct the message $x_{R}$ for the transmission in the third phase. Furthermore, the relay node simultaneously processes and forwards the received signals using the harvested energy.

In the third phase (Broadcasting phase, $\mathrm{BC}$ phase), the relay node broadcasts the signal to the receiver. The received signal $y_{D}$ at the destination terminal $S_{b}$ can be presented as:

$$
y_{D}=\sqrt{P_{R}} g x_{R}+n_{D}
$$

where $g$ denotes the channel coefficients between the relay and the destination terminal. $P_{R}$ is the transmit power for the relay node. The additional Gaussian noise at the destination terminal is $n_{D} \sim \mathrm{CN}(0,1)$. Then, the data rate of the destination terminal can be expressed as:

$$
r_{D}=\alpha_{3} \log _{2}\left(1+P_{R}|g|^{2}\right)
$$

where $\alpha_{3}$ is the broadcasting duration of the relay node in the third phase.

Until now, the rate in DF cooperative network with energy harvesting can be presented as:

$$
r=\min \left(r_{R}, r_{D}\right)
$$

It is noted that the transmission duration of each phase is flexible. The time ratios of all the phases should be maintain the following constraint:

$$
\alpha_{1}+\alpha_{2}+\alpha_{3}=1
$$

\section{Problem Formulation and Optimal Resource Allocation in Cooperative Network}

In this section, we investigate the optimal power control and time resource allocation among three phases to maximize the system throughput. At the energy 
harvesting relay node, the scavenged power from the first phase should afford to broadcast the signal which is received in the second phase to the receiver during the third phase. The optimization problem can be formulated as follows:

$$
\begin{array}{cr}
\underset{\alpha_{i}, P_{i}}{\operatorname{maximize}} & r=\min \left(r_{R}, r_{D}\right) \\
\text { s.t. } & \alpha_{1}+\alpha_{2}+\alpha_{3}=1 \\
& \alpha_{3} P_{R} \leq Q_{R}=\alpha_{1} \zeta P_{1}|h|^{2} \\
& \alpha_{1} P_{1}+\alpha_{2} P_{2} \leq Q
\end{array}
$$

It is noted that the transmission duration for each phase is the variable parameter to be optimized. It is not suitable to adopt the power constraint for this system, since the total energy consumption would be different for different transmission duration. Therefore, an energy constraint is proposed for the transmitter. It can maintain a fair comparison with other traditional algorithms.

The optimization in (8) is a non-convex problem, which in general has intractable complexity due to the variables' multiplied nature. However, we proposed a method to find an optimal solution via built on the reformulation of (8) into a convex optimization problem and strong Lagrange duality.

It is proposed to substitute:

$$
Q_{1}=\alpha_{1} P_{1} ; Q_{2}=\alpha_{2} P_{2} ; Q_{3}=\alpha_{3} P_{R}
$$

into the objective function and constraints of the problem (8). This reformulation does not change the original problem. Then the optimization problem can be reformulated as follows:

$$
\begin{array}{cc}
\underset{\alpha_{i}, Q_{i}}{\operatorname{maximize}} & r=\min \left(\begin{array}{l}
\alpha_{2} \log _{2}\left(1+\frac{Q_{2}}{\alpha_{2}}|h|^{2}\right), \\
\alpha_{3} \log _{2}\left(1+\frac{Q_{3}}{\alpha_{3}}|g|^{2}\right)
\end{array} \text { s.t. } \quad \alpha_{1}+\alpha_{2}+\alpha_{3}=1\right. \\
& Q_{3} \leq Q_{R}=\zeta Q_{1}|h|^{2} \\
& Q_{1}+Q_{2} \leq Q
\end{array}
$$

It is noted that the objective function in (10) is concave respective $\{\boldsymbol{\alpha}, \boldsymbol{Q}\}$. Since $\alpha_{2} \log _{2}\left(1+\frac{Q_{2}}{\alpha_{2}}|h|^{2}\right)$ and $\alpha_{3} \log _{2}\left(1+\frac{Q_{3}}{\alpha_{3}}|g|^{2}\right)$ are the perspectives of the concave functions $\alpha_{2} \log _{2}\left(1+P_{2}|h|^{2}\right)$ and $\alpha_{3} \log _{2}\left(1+P_{R}|g|^{2}\right)$ respectively [11]. It is also noted that the minimum of two concave functions is a concave function. Furthermore, since all the constraints are convex sets and there are obvious feasible points, Slater's condition is satisfied [12]. Hence, the convex optimization problem (10) has zero duality gap. It is suggesting that a globally optimal solution can be found in the Lagrange dual domain.

The Lagrange function can be given as: 


$$
\begin{aligned}
& \mathrm{L}(\boldsymbol{\alpha}, \mathbf{Q})=\min \left(\begin{array}{c}
\alpha_{2} \log _{2}\left(1+\frac{Q_{2}}{\alpha_{2}}|h|^{2}\right), \\
\alpha_{3} \log _{2}\left(1+\frac{Q_{3}}{\alpha_{3}}|g|^{2}\right)
\end{array}\right)-\mu_{a}\left(Q-Q_{1}-Q_{2}\right) \\
& -\mu_{b}\left(\zeta Q_{1}|h|^{2}-Q_{3}\right)-\mu_{c}\left(1-\alpha_{1}-\alpha_{2}-\alpha_{3}\right)-\lambda \boldsymbol{\alpha}^{T}-\boldsymbol{\beta} \boldsymbol{Q}^{T}
\end{aligned}
$$

where $[\boldsymbol{\mu}, \lambda, \boldsymbol{\beta}]$ are the Lagrange multipliers associated with the energy constraints and time ratio constraints. The dual function is given as follows:

$$
\begin{gathered}
\mathrm{G}(\boldsymbol{\mu}, \boldsymbol{\lambda}, \boldsymbol{\beta})=\max _{\boldsymbol{\alpha}, \mathbf{P}} \mathrm{L}(\boldsymbol{\alpha}, \boldsymbol{Q}, \boldsymbol{\mu}, \boldsymbol{\lambda}, \boldsymbol{\beta}) \\
\text { s.j. } \quad \alpha_{1}+\alpha_{2}+\alpha_{3}=1 \\
Q_{3} \leq Q_{R}=\zeta Q_{1}|h|^{2} \\
Q_{1}+Q_{2} \leq Q
\end{gathered}
$$

The above maximization of the Lagrange function can be solved out by first optimizing the energy allocation given $\boldsymbol{\alpha}$ fixed. Using the KKT conditions, the optimal energy allocation can be given as:

$$
\begin{aligned}
& Q_{1}=\frac{Q_{3}}{\zeta|h|^{2}} \\
& Q_{2}=\frac{\alpha_{2}}{|h|^{2}}\left[\left(1+\frac{Q_{3}|g|^{2}}{\alpha_{3}}\right)^{\alpha_{3} / \alpha_{2}}-1\right]
\end{aligned}
$$

where $Q_{3}$ is positive root of the following function:

$$
\frac{Q_{3}}{\zeta|h|^{2}}+\frac{\alpha_{2}}{|h|^{2}}\left[\left(1+\frac{Q_{3}|g|^{2}}{\alpha_{3}}\right)^{\alpha_{3} / \alpha_{2}}-1\right]=Q
$$

Next, we consider the issue to maximize the Lagrange function over $\boldsymbol{\alpha}$. From the objective function, it is noted that $\alpha_{1}$ did not play a role of maximizing the original problem. It means that, in order to maximize the system rate, the transmitter would operate in the regime of infinite power in the EH phase due to $\alpha_{1} \rightarrow 0$, which cannot be implemented with practical power amplifiers. Hence, a more feasible region for transmit power in each phase is obtained by adding peak transmit power constraints: $P_{1}, P_{2}, P_{R} \leq P_{\text {peak }}$.

Apparently, the optimal $\alpha_{1}$ can be given as:

$$
\alpha_{1}=Q_{1} / P_{\text {peak }}
$$

Then $\alpha_{2}, \alpha_{3}$ should maintain the constraints:

$$
\alpha_{2}+\alpha_{3}=1-Q_{1} / P_{\text {peak }}
$$

Until now, there are only two variables in the reformulated problem. We can use the Golden-section or interior point method to find the optimal solution.

\section{Simulation Results}

In this section, the performance of optimal resource allocation in cooperative network would be investigated. For comparison, we adopt tworeference methods as introduced in [7] and [9]. The communication protocols of the two articles semi fix the time ratio among three phases. They are given as in Figure 2. 


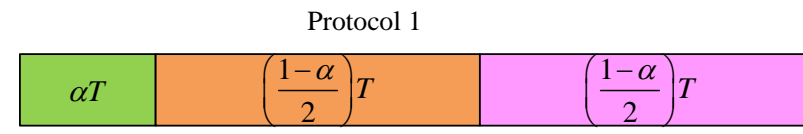

Protocol 2

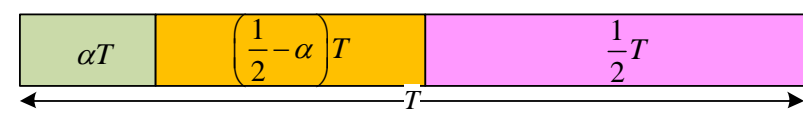

EH phase IT phase BC phase

Figure 2. Time Ratios for Three Phases in Two Transmission Protocols for Relay Network with Energy Harvesting

Supposed that all the channel coefficients are complex Gaussian with zero means and unit variances. Three nodes are distributed in a one-dimensional region. Denote the distance between transmitter $S_{a}$ and relay node as $d_{i}$ and for destination terminal $S_{b}$ as $1-d_{i}$. Suppose the large scale path loss factor $\phi=3$, the channel coefficients between relay and terminals would have additional large scale path loss $\Omega_{1}=d_{i}^{-3}, \Omega_{2}=\left(1-d_{i}\right)^{-3}$, respectively.

Firstly, we evaluate the performances of the system sum rate of all the mentioned methods. It is noted that the transmission duration in different protocol would be different. It is reasonable to adopt the energy constraint to evaluate the performance of all the mentioned methods. It is considered that transmitter and receiver could not both operate in the regime of infinite power in the EH time slot due to $\alpha \rightarrow 0$, which cannot be implemented with practical power amplifiers. Supposed that the maximum transmit power $P_{\text {peak }}$ is constrained with $P_{\text {peak }} \leq 4 Q$. Figure 3 shows that the proposed method can achieve higher throughput compared with two other methods under different transmit energy with $d_{i}=0.2$. It is because that the proposed method using more flexible time ratio to balance the channel fluctuation between two hops.



Figure 3. System Sum Rates Under Different Transmit Energy with $d_{i}=0.2$ 


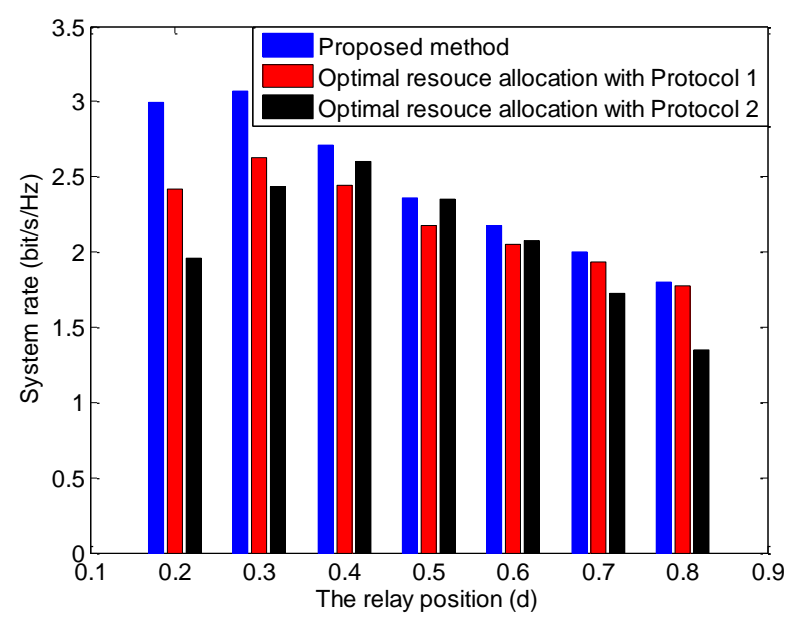

\section{Figure 4. System Sum Rates Under Different Relay Position with} $Q=20 \mathrm{dBmw}$

Next, we investigate the system performance with different relay position. Figure 4 shows that the proposed method is always outperform than other two methods with different relay position. Moreover, the performance of protocol 1 is close to the proposed method when the transmitter is far away from the relay node. And the performance of the protocol 2 is near to the proposed method when the relay node is in the middle of two source terminals. It is due to that the transmit power is constrained by the peak power $P_{\text {peak }}$, there is a compromise between the channel condition and the time occupation of each hop.

\section{Conclusion}

In this paper, we investigate the optimal energy control and time allocation in wirelesspowered relay network. Different from the traditional transmit power constraint, we adopt an energy constraint to formulate the optimization problem. It is more reasonable and fair to evaluate system performance in energy harvesting cooperation system. It is due to that the occupation time for transmitter in different transmission protocol would be different. The total energy consumption in a whole transmission period would be variety if the power constraint adopted. We proposed to use the energy constraint to investigate the optimal resource allocation scheme in wireless-powered relay network. Moreover, it is demonstrated that the optimization problem is convex. The efficient algorithm is derived by using convex optimization technology. Simulation results shows that the proposed method can get higher system throughput by applying more flexible time ratios to balance the channel condition and time occupation for each phase.

\section{References}

[1] Krikidis, S. Sasaki, S. Timotheou and Z. Ding, "A Low Complexity Antenna Switching for Joint Wireless Information and Energy Transfer in MIMO Relay Channels", IEEE Transactions on Communications, vol. 62, no. 5, (2014), pp. 1577-1587.

[2] A. Kurs, A. Karalis, R. Moffatt, J. D. Joannopoulos, P. Fisher and M. Soljacic, "Wireless power transfer via strongly coupled magnetic resonances", Science, vol. 317, (2007), pp. 83-86.

[3] U. Olgun, C. C. Chen and J. L. Volakis, "Wireless power harvesting with planar rectennas for 2.45 ghzrfids" Proceedings IEEE URSI Int. Symp. Electromagnetic Theory (EMTS), (2010), pp. 329-331.

[4] M. Mi, M. H. Mickle, C. Capeli and H. Switf, "RF energy harvesting with multiple antennas in the same space", IEEE Antennas and Propagation Mag., vol. 47, (2005), pp. 100-106.

[5] L. R. Varshney, "Transporting information and energy simultaneously", Proceedings IEEE Int. Symp. Inf. Theory (ISIT), (2008), pp. 1612-1616. 
[6] X. Zhou, R. Zhang and C. K. Ho, "Wireless information and powertransfer: Architecture design and rate-energy tradeoff", IEEE Transactions on Communitions, vol. 61, no.11, (2013), pp. 4754-4767.

[7] A. A. Nasir, X. Zhou, S. Durrani and R. A. Kennedy, "Relaying Protocols for Wireless Energy Harvesting and Information Processing", IEEE Transactions on Wireless communications, vol. 12, no. 7, (2013), pp. $3622-3636$.

[8] C. Huang, R. Zhang and S. Cui, "Throughput maximization for the Gaussian relay channel with energy harvesting constraints", IEEE Journal on Selected Areas in Communications, special issue on theories and methods for advanced wireless relays, vol. 31, no. 8, (2013), pp. 1469-1479.

[9] Z. Ding, S. M. Perlaza, I. Esnaola and H. V. Poor, "Power allocation strategies in energy harvesting wireless cooperative networks", IEEE Transactions on Wireless Communications, vol. 13, (2014), pp. 846-860.

[10] I. Krikidis, S. Timotheou and S. Sasaki, "RF energy transfer for cooperative networks: data relaying or energy harvesting”, IEEE Commun. Lett. vol. 16, (2012), pp. 1772-1775.

[11] M. Hajiaghayi, M. Dong and B. Liang, "Jointly Optimal Channel and Power Assignment for Dual-Hop Multi-channel Multi-user Relaying”, IEEE J. SELECT. AREAS COMMUN. vol. 30, no. 9, (2012), pp. 1806-1814.

[12] S. Boyd and L. Vandenberghe, "Convex Optimization”, Cambridge University Press, (2004). 